\title{
The Process of Formation of a Single Star System for Gravitational Compression of a Gas-dust Cloud in Outer Space
}

\author{
Alexey Belyaev \\ Independent Researcher, Russia
}

Copyright@2019 by authors, all rights reserved. Authors agree that this article remains permanently open access under the terms of the Creative Commons Attribution License 4.0 International License

\begin{abstract}
In this paper it is shown that in the process of formation of a single star system favorable conditions are created for the appearance of the planetary system due to the collision of the oppositely moving condensed dust zones from the newly born star and falling onto it from the periphery. A hypothetical version of the possible structure of the core of a single star born in a rarefied gas-dust cloud, explaining the cause of the mismatch of the axis of rotation of the star and the dipole magnetic field, is proposed. An assumption is made about the causes of the appearance of a directed orbital motion of planets that are not related to external perturbations of the initial gas-dust cloud.
\end{abstract}

Keywords Gravity, a Rarefied Gas-dust Cloud, the Birth of a Single Star, Separation of Heavy Elements, the Formation of Planets and Asteroids

\section{Introduction}

At the present stage of evolution of the universe, gas-dust clouds in outer space form as a result of emissions or explosions of stars later generations. If the initial mass is sufficient the process of gravitational compression of such clouds ends with the birth of new stars. For example, you can get acquainted with the modern basics of the structure and evolution of stars in the work by Pravilnik [1].

In article [2] by Belyaev, the physical picture of the compression process of a dust cloud was considered using a chain of logical cause-effect stages. In the article, the term "dust cloud" was based on a broader principle than the conventional one, which made it possible to include of different types of systems in the subject matter. The main feature of the dust cloud in this paper is that when colliding with each other the particles that make up the cloud of dust consolidate their masses. The generalized approach made it possible to take a case in which galaxies were "dust particles" and show the premature introduction of the new category of "dark energy" into physics (the accelerated dispersal of galaxies in the universe can be described by classical gravity). Furthermore, the usual dust clouds considered by astrophysics are also a special case of a generalized dust cloud. The main result of studying the principles of compression of a generalized dust cloud, on which this work is based, is that gravitational forces compress dust particles of a cloud in outer space in such a way that the accumulating substance has the greatest density at the periphery. The inversion of the density, when the density becomes maximal in the central zone, occurs only with the appearance of short-range repulsive forces continuously distributed throughout the volume of the cloud.

Such an undeniable position, unusual for theoreticians, about the distribution of density of a dust cloud which self-contracts due to gravitational forces in the process of its compression (rather than at the end of the process) is not only an interesting fact in itself, but also serves as a key link in the initial stage of separation of heavy elements, even before the stage of formation of the planetary belt of a single star, at which separation develops and terminates. The stage of forming the planetary belt is initiated by the collision of a peripheral densified layer moving towards the center with an inner layer also densified but having the opposite direction of movement from the center. This work is devoted to the consideration of such new schemes of gravitational interactions during the spontaneous compression of a gas-dust cloud, including the study of the features of the formation of a star and a planetary belt in the vicinity of a burning star and the causes of the appearance of the considered physical processes are analyzed.

The goal set in this paper is to identify the main characteristic features of the process of gravitational compression in the space of a rarefied gas-dust cloud on the basis of a schematic description of the resulting physical processes. 
Many star systems formed as single isolated systems. The penultimate section of this paper contains comments on why our solar system can be attributed to a single star system. The main task set in this paper will be the identification of general principles for the evolution of single stellar systems.

First, the final stage of the Kelvin contraction that end with the birth of the star, will be considered. Emphasis will be placed on the physical principles that determine the directions of emerging processes in the formation of the nucleus of a compressible rarefied gas-dust cloud.

After that the topic of formation of planets and asteroids will be considered. The most pressing issue of this topic is the separation of heavy elements. Even until now there is no clear understanding of what physical principles could make it happen. Currently we have nothing but weighted assumptions. The causal analysis of the emerging physical phenomena will make it possible to explain the reason for the appearance and essence of the separation mechanism in visual-schematic images, but without decoding and detailing the resulting processes. The description of new physical effects in the formation of planetary nuclei is beyond the scope of this paper and will be dealt with in a separate subsequent publication.

Within the framework of this paper, an assumption will be made about the causes of the appearance of a mechanical rotation of a single star and the appearance of its dipole magnetic field, the axis of which does not coincide with the axis of the rotation. In addition, the assumption of the cause of the twisting of the substance, resulting in the formation of a planetary disk, of the stellar system in a single direction will be made. The twisting is caused not by external perturbations, but by the kinetics of the internal processes of a compressed rarefied gas-dust cloud.

\section{Methodology}

Another paper by Belyaev [3] touches upon the topic of the methodological approach to the construction of new physical and natural-science theories in addition to the analytical description of the evolution of stars.

Until the beginning of the twentieth century, any new natural science theory was required not only to be mathematically justified, but also to be explained in visual images based on the laws of Newtonian mechanics, even when considering unobservable hypothetical ether. The fact is that all the basic physical concepts, such as strength, energy, work, were based and, as unexpected as it might seems for a modern physicist, continue to be based on the recognition of the principle of inertia and the laws of Newtonian mechanics applicable only to corpuscles (the emergence of new directions of physics, beginning with thermodynamics did not change the basic physical concepts introduced earlier, but only with some success adapted them for new conditions of consideration). Many other basic physical concepts, including temperature, which is widely used in natural sciences, are also inextricably linked with the notion of "corpuscle". However, scientists have become so accustomed to basic terms that they often forget to realize auxiliary conclusions made in the course of their reasoning in the physical context. For example, today it is asserted that the Universe which has been born, even before baryogenesis, had a high temperature. Nevertheless, no scientist can explain what the temperature in the spatial zone is, in which there is no matter (and has never been before). For example, it is only possible to start a discussion about the temperature of electromagnetic radiation with the notion of "absolutely black body". The topic of the erroneous use of physical terms, without a deep awareness of their physical essence, can be developed more broadly. For example, the entrenched use of the term entropy that has taken root in practice. However, this topic is not the subject of this work. The topic being discussed is the application of the accepted and mandatory methodology in the XIX century for all natural sciences. A methodological approach that attempts to explain new physical phenomena using Newtonian mechanics has been called a "mechanism" by the philosophers.

However, quantum mechanics turned elementary particles from corpuscles into a hybrid "corpuscle-wave", artificially combining the theory of waves and corpuscles that are fundamentally incompatible with each other, furthermore, the theory of relativity deprived us of the concepts of absolute time and potential energy. Therefore, the mechanistic approach based on Newton's laws lost its universality. Moreover, mathematics came to the foreground, and even a new branch of physics, "theoretical physics", was formed, in which abstract mathematical models with varying degrees of approximation are based on physical principles, and not vice versa. After undermining the universality of logic, which is based on visual images and allows theorists to create mental films that track causal chains of described physical phenomena at a figurative level, such a methodological approach and view of the surrounding world was re-named "common sense", and it was asserted that it should not be trusted. Common sense has become less respected not only in physics, but in all natural sciences.

The main problem of the process cognition in the framework of natural sciences at present is not that the mechanistic approach more and more often becomes unhelpful and thus ceases to be useful, apart from some theoretical directions that have survived without significant changes since the 19th century; but forms a complete disregard for the complex analysis with the construction of causal relationships on physical principles. Main point being discussed are the "physical" principles derived from 
experimental observations, and not as a result of abstract theoretical research.

In work [3] it is explained that common sense still remains relevant and cannot be forgotten. For example, in this work, the misinterpretation of the fate of light gas clouds self-contracting in space, which are supposedly able to change from self-heating to self-cooling, is demonstrated in terms of common sense. In particular, cooling red and brown dwarfs cannot exist. In [3] it is also proposed to use common sense to understand the conclusions obtained in the framework of other approaches, for example, to comprehend the essence of the physical category "degenerate system".

As mentioned above, in work [2], common sense has arguably raised the fundamental question of the prematurity of introducing the theory of a new category of "dark energy". In the work of Belyaev [4], another fundamental question was discussed on the basis of common sense: the incorrectness of recognizing cosmic microwave background as a relic. The results of $[2,4]$ from independently revealed that our Universe is surrounded by a massive homogeneous halo. In the work of Belyaev [5], common sense was used to demonstrate the incorrectness of the Big Bang model and the cosmological inflation theory (instead, principles were proposed for constructing a new model for the birth of the Universe). In Belyaev [6], common sense was used to show the incorrectness of the method used today for applying the laws of classical gravity near extended material objects and inside them. In addition, the principle of the formation in the largest galaxies of a heavy halo, consisting of a substance invisible from Earth in the electromagnetic radiation of a substance is explained in this work, and the character of the spatial distribution of mass in the halo is specified. In fact, although this was not directly stated, a preliminary indication was given in [6] that it was premature to introduce into the theory a new category of "dark matter", which forms the hidden mass of the halo of galaxies. If in work [6] it is explained how the hidden mass could concentrate on the periphery of the galaxy with the almost complete absence of matter outside the central visible zone of the galaxy, then the possible variants of the mechanisms for the creation of the hidden mass are indicated in [3]. In addition, Belyaev [7], also on the basis of common sense, showed the possibility of the existence of a currently unknown mechanism for the compression of a gas cloud in outer space which is accompanied by self-cooling (a real cloud in outer space is always an uninsulated system). Such a mechanism could have made a significant contribution to the overall formation of the hidden mass. The question of the formation of the hidden mass will be discussed in more detail in the framework of the modeling of the corresponding stage of the evolution of the Universe in a separate publication.

This paper, in no way calls for the recognition of "common sense", based on Newton's mechanics, as universal. Newtonian mechanics cannot cope with many of the tasks of life. The focus is on the need for a detailed analysis with an required mindfulness of causal relationships. Since only the Newtonian mechanic and everything built on its base ${ }^{1}$, is actually recognized, theorists first need to check the applicability of the mechanistic approach, reinforcing the exposed patterns by mathematical justification, and only then proceed to abstract theories. It is completely unacceptable to use new categories that have no physical meaning, such as "black hole", "cosmological inflation", "dark energy", "dark matter", etc., not as a temporary hypothesis or "black box", but as fundamental elements of theories. The effectiveness of the "preliminary fitting of mechanics" approach has once again been demonstrated by this work, which has made it possible to uncover many new things that were unknown before. The entire analysis of this work is based on common sense.

\section{General Theoretical Principles of the Initial Stage of the Process of Gravitational Compression of a Rarefied Gas-dust Cloud}

Gravitational forces are in direct proportion to masses of interacting objects and inversely proportional to square distance between them, they are subject to the principle of superposition (total gravitational influence on trial mass can be divided into separate influences from each surrounding object).

If we take two stationary particles, being in emptiness at a distance of $R$, they will begin to draw towards one another, and in some time will converge until they colide. If the mass of each particle is multiplied by $n$, the force of their interaction, depending on the current distance between them, will be multiplied by $n^{2}$, and therefore, the acceleration of each particle, changing in time, will also be multiplied by $n$, so the time of convergence of the two more massive particles will be decreased by $n^{2}$. Meanwhile, the energy of gravitational contraction will be entirely transferred into kinetic energy of colliding particles.

Clouds of equal particles will act in the same way: heavy particles will converge to the central zone of their cloud faster, then light particles to the central part of their cloud. The energy of gravitational contraction of the particles colliding in the central zone will transform into internal energy of matter, both in case of gas, when the particles

1 Special and general theories of relativity, quantum mechanics and quantum field theory are abstract theories that are not realized in visual images, but only obey the rules established by their fundamental principles. But other theories that can replace them, have not yet been created. 
colliding with each other in the central part of the cloud will resiliently repel from each other, just like in the case of dust, when the particles colliding with each other in the central zone of cloud will unite into a single mass.

If we change the mass of only one particle in the model of two separate gravitationally interacting particles located in emptiness at distance $R$, the change of force of joint gravitation attraction will be directly proportional to the change of mass of this particle, but its acceleration will remain unchanged. For this reason all objects fall to the surface of the Earth with an equal acceleration, no matter what mass they have. This means that if you create a homogeneous cloud of light and heavy particles that are stationary relative to each other at the initial moment of time, and position the cloud in an emptiness, then the compression rate under the influence of gravitational forces of mutual attraction of the interacting particles of the cloud towards the center, heavy particles will not have the advantage, despite their faster compression in a clean, pure cloud compared to the compression of the same cloud, but consisting of lighter particles. Thus, the multicomponent cloud will be compressed without separating the flows: all particles of each selected sphere of the gas-dust cloud will move to the center of mass of the cloud synchronously to each other, regardless of their mass.

In this case, a cloud, in which at the initial moment of time all particles that make up the cloud, including gas, are conventionally fixed relative to each other is being considered. Such a condition can, with some assumption, be approximately observed in practice in the case of a rarefied and cold cloud. The main criterion for such an assumption is that the mean free path between collisions of particles of a cloud between themselves is less or comparable with the size of their radial displacement caused by gravitational attraction.

At the initial stages of the Kelvin compression of a rarefied gas-dust cloud, there are no significant differences between its compression and the compression of a purely gas rarefied cloud, since heavy particles do not have the advantage of moving to the center. It is very important to note the fact that the mechanism promoting the twisting of the elements of the gas-dust cloud around the center of mass of the cloud in arbitrary directions does not generate gravitational attraction forces. Moreover, there are no prerequisites for the emergence of a preferential direction of the particle movement around the center of mass or around an arbitrary axis within the cloud, which is necessary to be able to explain the phenomenon of gravitationally compressible substance of a gas-dust cloud into a flat disk.

However, after a certain amount of time, differences in the compression of light and heavy particles tend to become increasingly apparent. First, let us consider the characteristic features of the spontaneous compression of the gas and dust subsystems, which manifest themselves in the central zone of the cloud.

\section{Formation of a Nucleus in a Rarefied Gas-dust Cloud}

Modern astrophysics considers the gas-dust cloud according to the principle as if it were a pure gas cloud in which convective processes take place throughout the entire central volume of a future star. Dust is an integral part of gas and is considered only as an absorber of electromagnetic energy, which allows the gas-dust cloud, which in this case is a dusty gas, to more effectively increase its temperature and pressure in the central zone compared to the dust-free gas.

The mass of dust in a gas-dust cloud is usually only about $1 \%$ of the mass of the carrier gas. Therefore, the adopted approach is completely valid for mass formation of stars in dense galactic disks. In this case, however, the formation of a single star system is being considered. In this regard, to model a process close to reality, one should consider, firstly, a variant of a rarefied cloud and, secondly, an initially cold cloud. To be able to create a basic model, it should be assumed that the cloud at rest (not disturbed by convective processes). But with such initial conditions, the gas-dust system needs to be further divided into two sub-systems: gas and dust. It is unacceptable to consider a rarefied gas-dust cloud in the processes of formation of a single star system as a gas cloud. Gas, even if it is dusty, remains a gas. The result of evolution for a gas cloud will be completely different, compared to the two-component system with a relatively independent behavior of its subsystems.

For a calm rarefied gas-dust cloud consisting of two subsystems (gas and dust), there will be no turbulization of dusty gas in the central zone. Instead, the following processes will take place. Heavy particles will begin to unite with each other in the central zone, without creating pressure that impedes the movement of particles to the center of the cloud. And the pressure of light gas particles (hydrogen and helium, that make up our Universe) in the central zone will begin to grow in its subsystem to such an extent that it impedes the movement of gas particles from the periphery towards the center. In addition, the heavy elements, until they merge in the central part of the gas cloud, will also increase the gas pressure in the center of the cloud, transferring part of their kinetic energy to the elements of gas. However, this pressure will not be enough to prevent the penetration of heavy particles of dust and heavy chemical elements into the central zone. Therefore, from the very first stages of compression, a solid heavy core will begin to form in the center of the future star, and the zone surrounding the core of the static gas with increased pressure, not disturbed by convective processes, will be formed more actively than for a pure gas cloud.

This is an indisputable and obvious phenomenon, but it is not currently taken into account by modern astrophysical models. It is so obvious that a special justification for the inevitability of the formation of a heavy nucleus even 
before ignition of thermonuclear reactions is not required. Mathematical support and model building will be required when developing a rigorous theory of the gravitational compression of a gas-dust cloud. But mathematics is not required to substantiate the inevitability of the formation in the center of a calm rarefied gas-dust cloud of a heavy nucleus from the very beginning of the process of compression. It is only necessary to understand that a rarefied gas-dust cloud is not a dusty gas, but a mixture of gas and dust, which should be considered separate subsystems of a single gas-dust cloud.

Today, for compressible gas and gas-dust clouds, it is assumed that due to the increase in density and pressure, the compression of the central part occurs more actively than the compression of peripheral areas. However, for a rarefied gas-dust medium, the opposite tendency is obvious: more active compression must occur at the periphery, and not at the center of the cloud. This is because the peripheral particles acquire the greatest acceleration in the direction of the center of mass as a result of the action of gravitational forces and it becomes possible for them to catch up with the particles moving in front of them. At the same time, the laws of statistics, which transmit the increments of the kinetic energies of peripheral particles to the central zones, do not work, and the clearly expressed general direction of the movement of peripheral particles towards the center remains.

Today it is believed that with an increase in the density of the nucleus, when it reaches about $1 \%$ of the total mass of the gas-dust cloud, it becomes hydrostatically resistant to gravitational compression, as if becoming the progenitor of a star, i.e., becoming a proto-star. The proto-star continues to gain mass and increase its volume not as part of the cloud, but as an independent object, due to the accretion of the remaining part of the gas-dust cloud spinning by the time the proto-star appeared. There are no specific reasons for the appearance of spinning. The general direction of the revolving appears due to the violations of central symmetry of moving convective jets, that are attracted to the center of mass of the cloud. At the same time, the appearance of convection itself, including the appearance of violations of central symmetry, arose solely due to external disturbance factors that initially affected the gas-dust cloud during the initialization of its gravitational compression.

It is obvious that modern reasoning, which isolate a proto-star into an independent object, is erroneous. Erroneous for the reason that the density of the substance around the proto-star is still substantial. Therefore, it is unacceptable to neglect the mass located between the proto-star and its external outer concentric spherical layer, for the elements of which the gravitational attraction to the central zone of the cloud is being studied. In addition, it is also impossible, as shown in [6], to neglect the mass of the outer layers for the spherical layer under consideration. It is not the proto-star that forces an arbitrarily chosen outside element of the cloud to move towards it, but all the elements of the cloud, without exception, acting on the principle of superposition on the selected element, accelerate this element to a common center of mass. And the pressure inside the proto-star continues to grow not according to the law, which is determined by the mass of the proto-star, but according to the laws of compression of the entire cloud.

Modern astrophysics assumes that over time, the main part of the dust of a gas-dust cloud is absorbed by the proto-star. As a result, the proto-star is released from the optical veil and flashes in the infrared radiation emanating from the dense dust cover enveloping it. The farther away from the proto-star is the gas, the less dusty it is.

Indeed, the proto-star is released from the veil and becomes observable in infrared radiation (from astronomical observations it does not just become observable, it "ignites"). However, this is not due to the absorption of the main part of the dust of a gas-dust cloud by the proto-star, but solely due to the separation of dust into two compacted zones: internal and peripheral. Moreover, the bulk of the dust is contained in the peripheral zones (!), which, due to their large geometric dimensions (the volume of the elementary spherical layer of the ball depends on the square of the distance to this layer), tend to be transparent for radiation. In this case, the dust of the middle zone of the cloud can even acquire reverse acceleration towards the periphery (see [2]), thus, increasing the concentration of dust in the peripheral zone and reducing it in the middle zone.

Thus, the formation of a heavy solid core in a gravitationally contracted rarefied and calm gas-dust cloud is inevitable. The formation of the nucleus should take place in three stages. In the first stage, acts of frontal collisions of heavy particles cause a solid core seed to be formed. The next stage will see the formation of a compacted accretion zone with pseudo-orbital movement of heavy particles around the formed solid core. And the third stage will bring about the completion of the process of formation of a solid core and its accretion of unused heavy particles. Moreover, it is hypothetically possible, that at the third stage the formed solid core will turn into a degenerate structure. Let us consider the three stages of the formation of the nucleus that will be rationally associated with the general stages of the evolution of the entire rarefied gas-dust cloud in general, which will be done in the next chapter, in more detail.

When a pure gas cloud is gravitationally compressed, a decrease in its geometric dimensions is primarily accompanied by a redistribution of pressure inside the cloud due to operation of statistical laws seeking to redistribute the internal energy of the thermodynamic system to an equilibrium state for the entire system (in the absence of a long-range field inside the system, particularly, the gravitational field, the density of internal energy due to the laws of statistics must be equalized over the entire 
volume of the thermodynamic system). In the absence of collapse, i.e., when a gravitational contraction occurs without a concomitant significant violation of the laws of statistics (and the gravitational field always inevitably violates the laws of statistics), the pressure increases to a greater extent in the central zone of the compressing gas cloud.

Unlike a gas cloud, a pure dust cloud is not characterized by the chaotic motion of its particles. The gravitational compression of a dust cloud is primarily characterized by the radial movement of each of its particles towards the center of mass, providing a "drainage" for heavy particles arriving at the center due to their agglomeration. Due to the appearance of a solid nucleus, dust particles are ejected from the dust cloud system, which, for this reason, turns into an open system.

In a quiet rarefied gas-dust cloud, at the initial stage of its compression, the gas and dust subsystems are practically independent of each other and should manifest themselves as pure clouds: the gas subsystem will not interfere with the formation of a solid core of heavy particles sticking together at the center, and heavy particles will not hinder the formation of a static gas cloud in the central zone with continuously increasing pressure. Similar processes will be seen at the periphery of the cloud, but in a very weak form: dust particles at the periphery will coarsen, and gas particles will increase their static pressure in coordinate systems rigidly connected with areas of the masses moving towards the center of the masses. This is the first stage of the formation of a nucleus under the spontaneous gravitational compression of a rarefied gas-dust cloud in outer space.

However, after a while, the gas and dust subsystems will become more sensitive to each other. Primarily this will happen in the central zone of a gas and dust cloud. The gas surrounding the solid core, due to the increase in its pressure and density, will begin to deflect heavy particles falling on the core thus leading them to parabolic trajectories. The newly emergent heavy and gas particles arriving from all spatial sides will begin to transfer diverging particles to pseudo-orbital trajectories. "Pseudo-" because their real velocities will exceed normal orbital velocities. Furthermore, the orbits occupied by particles with a certain kinetic energy will not be determined by the mass of the solid core. Moreover, the heavy particles will "break off" from these orbital trajectories in the direction of the solid core, making it more compact and increasing its mass. The average statistical result will bring about a formation of a vortex compacted zone of heavy particles, falling on the seeding core and accelerated not only by the seed field of gravity, but also by relatively dense layers of the gas subsystem, which temporarily supplement the seed core mass, but that are subsequently displaced by falling heavy particles. At the same time, the gas, previously washing the solid core, displaced from the central zone, continues to increase its pressure not only due to the processes in its gas subsystem, but also more actively due to dissipation of all newly arriving heavy particles (the kinetic energy of the arriving heavy particles exceeds the kinetic energy of the particles in the gas subsystems, both gravitationally attracted from the periphery and moving together with the falling heavy particles, and those already within the central zone). A summary of the second stage of the formation of a nucleus of a rarefied gas-dust cloud is the appearance of a high-energy zone of accretion of heavy particles in the vicinity of a solid core and an intensive increase in the density, temperature and pressure of the gas around the central zone. As for changes in other parts of the cloud, at the second stage a compacted peripheral zone of a compressing gas-dust cloud and a rarefied zone in its middle part should have already formed.

After some time, the increase in the pressure and temperature of the gas in the central zone will be favorable for the start of thermonuclear reactions, which release large portions of energy for each fusion event of the interacting atomic nuclei. A third and final phase of the formation of a nucleus of a rarefied gas-dust cloud will begin. The access of new portions of heavy particles to the central zone should stop, and their accumulation will begin at the periphery of the formed nucleus. Since the ignition of the star, i.e., from the moment of the appearance of self-sustaining thermonuclear reactions, the mass of the nucleus will remain constant. Under conditions of constancy of the mass, the structure of the nucleus should finally be formed. Moreover, hypothetically a very interesting event may arise: the complex composite structure of the central zone (nucleus and vortex zone of accretion) at the time of the first wave of thermonuclear reactions and before the moment of ignition of self-sustaining self-regulating thermonuclear reactions, will undergo a transformation, having turned into a degenerate structure. The theoretical possibility of its occurrence will be pre-evaluated at a qualitative level later on in this work.

The question of whether a nucleus with a degenerate structure can form in the gas-dust cloud at the stage of star formation is quite complex. In addition to creating a model for such a process, it will also be necessary to develop new theoretical provisions in which the formation of a white dwarf or neutron star takes place at the small seed center, which has a degenerate structure. Most likely, for the gas-dust cloud that forms the nucleus, which later becomes the central part of the new nucleus of a star being born, this can only be said about the structure with a degenerate electron gas. More precisely, the results of a rigorous and not a generalized analysis can determine whether a structure with a degenerate neutron gas can form. And the very conclusion about the formation of the Kelvin compression of a degenerate structure in the core of a self-contracting rarefied gas-dust cloud at the final stage is only permissible as a hypothetical assumption that requires 
theoretical verification. Hypothetically, it is also possible that a white dwarf will have a complex structure with a nucleus of a degenerate neutron gas and a shell of a degenerate electron gas.

However, the fact of the appearance of not a degenerate structure, but a heavy nucleus, which may or may not turn into a degenerate structure, is undeniable. The conclusion about the presence of an emerging single star in a self-contracting rarefied gas-dust cloud of a heavy solid core is new, indisputable and obvious, and does not require special proof.

\section{Separation of Heavy Elements and Formation of Planets}

The scheme for the compression of a rarefied gas-dust cloud in outer space presented above is not complete. In the first moments of gravitational compression initialization the gas and dust subsystems behave similarly, with the exception of the result obtained after mutual collisions of particles in their subsystems, however, over time, the differences in gravitational processes for these two subsystems begin to manifest not only in the central zone, but also on the periphery of the cloud.

As shown in [2], the frequency of particle collision during gravitational compression inevitably increases, furthermore, the increase in the frequency of collisions and an increase in the kinetic energies of particles mainly on the periphery of the cloud are initiated. However, for a thermodynamic system in which the laws of statistics work, for example, for a gravitationally compressing gas cloud, frequent elastic collisions of gas particles, as mentioned above, redistribute the gas pressure in such a way that its pressure counteracting the forces of gravity increases to a greater extent in the central cloud zone.

At first glance, it seems that at the second stage of compression, the gas-dust cloud should no longer be considered rarefied, and that it is erroneous to continue dividing it into relatively independent dust and gas components. The energy of the gas and dust particles accelerated by gravity in the peripheral and middle zones of the cloud will be transmitted through acts of their elastic collisions with gas particles into the central region of the cloud, increasing not only the gas pressure inside it, but also dusty gas pressure (gas-dust mixture). Furthermore, the dust particles of the inner zone can be increasingly connected to the chaotic motion inherent to the gas particles. Therefore, the separation of the gas-dust system into gas and dust components with the onset of the second stage of compression becomes incorrect. However, a closer examination shows that the first stage creates such initial conditions for the second stage, that the gas-dust mixture must still, despite the increase in the average density, be considered a rarefied gas, and the system should be further studied through the interaction of its two subsystems.
The trends that emerge are as follows. On the one hand, the laws of statistics begin to increasingly manifest themselves. However, the long-range gravitational impact violates the laws of statistics, increasing its destructive effect towards the periphery. But if the observers moving synchronously with layers of a gravitationally compressed gas-dust cloud measure pressure at their observation points and compare the results with the measurement of an observer resting relative to the center of mass of the cloud and measuring the pressure in the central zone sample, then the pressure of any sample from the middle zone will be lower than the pressure samples of the central zone. In other words, the static pressure in the central zone will be the highest. Quite a clear tendency emerges that one should speak not about the measured pressure of the gas subsystem, but about the pressure of the dusty gas.

On the other hand, despite the increase in gas pressure in the central zone, its particles are unable to disrupt the predominant movement of heavy particles toward the center. Firstly, heavy particles (heavy chemical elements and dust particles), when combined, increase their mass (the mass of particles of the dust subsystem is constantly growing), i.e., it becomes more difficult for gas to pick up dust. Secondly, over time, not only the pressure and temperature of the dusty gas in the central zone increase, but also the kinetic energies of gas and dust particles arriving from more distant radial distances (gravitational forces do increasing work on particle acceleration; in the first approximation, dust particles arriving from the periphery exceed the kinetic energies of the arriving gas particles in proportion to their masses). Moreover, the increase in the temperature of the inner zone is a consequence of an increase in the acceleration of particles arriving from the periphery, i.e., the kinetic energies of the incoming particles are on average higher than the kinetic energies of the particles in the central zone. The counteraction to the process of gravitational compression, namely, the increase in the pressure of the gas in the central zone, approaching the level necessary for restraining the energy of the gravitational forces, is primarily due to the increase of the density of the gas, and only then is it associated with an increase in temperature. Therefore, the tendency opposing the predisposition of combining the gas and dust subsystems into a single gas-dust system, which has the characteristic features of a pure gas system, is prevalent in the central zone of the gas-dust cloud. Thus, in the central zone, the dust subsystem of a gravitationally compressing rarefied gas-dust cloud at the initial phase of the second stage behaves, as in the first stage, differently from the gas subsystem, when the mutual influence of the subsystems on each other was almost completely absent.

By the beginning of the second stage, the dust subsystem also retains its independence at the periphery of the cloud. This occurs primarily due to the enlargement of dust particles. Moreover, the static gas pressure at the periphery also inevitably increases. 
However, in the middle zone, there is little difference in the behavior of the cloud in the first stage if we do not highlight the increase in the number of dust particles changing the speed of their radial movement from the direction towards the center to the direction towards the periphery as a special feature. Therefore, there will be virtually no confrontation of the two subsystems in the middle zone.

All this means that at the second stage of the formation of the core of a rarefied gas-dust cloud, the dust and gas subsystems of the cloud, due to moderate interaction, need to be considered independently of each other, as in the first stage (of course, "consider independently" - in principle; mutual influences will inevitably appear).

Summarizing the above, one can declare that the opposition of the dust subsystem to the complete subordination of the gas subsystem and the merging of subsystems into a single system, which continuously takes place in a gas-dust cloud, should be considered successful. Therefore, the measured pressure of the gas-dust cloud should be attributed to the pressure of the gas subsystem. The gas subsystem actively influences the dust subsystem. But the dust subsystem is characterized not by pressure, but by the kinetic energies of its constituent particles. Moreover, particles of the dust subsystem falling into the central zone of the cloud retain their superiority in magnitude of their kinetic energies over the kinetic energies of any particles of the central zone throughout the second stage of nuclear formation described in the previous chapter, and, as a result, the dust subsystem retains its individuality at this stage. It becomes rational to extend this to the stage of compression of the entire rarefied gas-dust cloud, generalizing the second stage of the formation of the nucleus to the stage of evolution of the whole gas-dust clouds in general.

So, the nature of the behavior of the dust subsystem, despite the active influence of the gas subsystem, retains the peculiarities of the generalized dust system described in [2]. As a result, dust will accumulate not only in the center of the cloud, but also at its periphery. Moreover, the accumulation of dust on the periphery will occur not due to accretion, but due to acts of collisions of dust particles having a radial component of their speeds as the predominant one. Over time, the acts of collisions of dust particles on the periphery of the cloud should increase their intensity and energy flow. In fact, a sufficiently active and effective consolidation and compaction of dust particles will begin to take place without disturbing the general direction of their radial movement towards the center. Perhaps, in this sense, the second stage of compression of the gas-dust cloud should be associated with the most important initial stage of separation of heavy elements, in which not only the formation of the discrete peripheral zone of the cloud, enriched with dust, takes place, and that not only a small part of the initial amount of dust is enriched, but so dust part of the entire gas-dust cloud, as well as the conglomeration of dust. Moreover, although the outflow of dust in the central zone retains its activity, it also turns into a discrete zone of increased dust density, primarily due to the influence of a usual geometric factor in the spatial distribution of the cloud. This additionally increases the significance of the process of the initial stage of separation of heavy elements, which begins at the second stage of the compression of a rarefied gas-dust cloud in space. Thus, the second stage of compression of a rarefied gas-dust cloud, which coincides with the second stage of the formation of the core of this cloud, is also connected with the start of the mechanism of spatial separation of heavy cloud elements between the peripheral and inner zones of the cloud, and the enrichment process of the two considered zones with dust is accompanied by its conglomeration inside these zones.

It is useful to note that in both dust-enriched zones there are still no stimuli arising for the initiation of the orbital motion of the elements of the dust subsystem, especially with a predominant orbital direction of motion. All the described processes occur at the Kelvin stage of compression of a gas-dust cloud.

After a certain time interval, the temperature and pressure of the gas washing the core and enriched with heavy elements will increase to a point where the gas will first turn into a plasma, and then nuclear reactions will be initiated in the plasma, i.e., the star will be lit. The start of thermonuclear reactions will form a new composite core around the existing solid central part, and completely stop the movement of heavy elements into the central zone, blocking the outflow of heavy elements from the gas-dust cloud system. Dust will begin to accumulate intensively around the new core of the star. The third stage of the formation of the core of a rarefied gas-dust cloud will begin.

After a certain time after the beginning of the third stage, intensive accumulation of dust on the periphery of the nucleus will cease, and the enlarged dust particles will begin to move away from the nucleus, overcoming the second cosmic velocity and forming the primary type of the emerging stellar wind. The mechanisms for the occurrence of stellar wind are described in numerous literature, including educational.

Initially, astrophysical models assumed that the emerging stellar wind was a key influencing factor for the launching mechanism of the process of planetary formation. However, modern astrophysical models, taking into account the data of newly obtained astronomical observations, believe that by the time a star ignites, the active processes of planetary formation are already at a maximum of their intensity, because the proto-star is already characterized by directional axial rotation, and there is already a practically flat protoplanetary disk is formed, in which the active the formation of planets takes place. Moreover, the protoplanetary disk, as it is assumed to date, consists of dust remnants, the main part of which 
are already absorbed by the proto-star. At the same time, the resulting stellar wind does not significantly affect the process of the formation of planets.

In this paper, a completely different mechanism of planetary formation is considered, dissimilar to the general directions in existing models, in which at a certain stage of the evolution of a star system two compacted dust zones must undergo a head-on collision with each other. If the appearance of a peripheral compacted zone, narrowing towards the center, for a rarefied gas-dust cloud, described by separate compression laws for its dust and gas subsystems, becomes apparent in the current presentation of the material, the intensity of the stellar wind for the creation of highly efficient accretion zone where the planets are formed, when the internal zone in its oncoming movement to the peripheral layer "stretches" into a stream of star wind, raises serious doubts.

Therefore, it is more likely, as will be seen from later reasoning, that the launch stage of self-regulating thermonuclear reactions, i.e., the star ignition stage, and the appearance of the stellar wind, is preceded by a small thermonuclear explosion, accelerating the main part of the solid particles of the inner zone towards the periphery. An explosion will create a wave of a scattering impacted zone. At the same time, the stellar wind, which is characterized by its continuity and moderate intensity even at the first stages of its occurrence, although a significant factor for the emerging planetary system of a newly born star, but it is, nevertheless, secondary. Moreover, as mentioned earlier, the moderate influence of the stellar wind on the processes of planetary formation is confirmed by new astronomical observations.

The efficiency of the process of planetary formation when it takes place according to the scheme indicated in this work is a result of the extinguishing of radial components of velocities of the already enlarged particles of the dust subsystem of the cloud. The collisions of solid fragments are accompanied by their full or partial assimilation with each other. The law of conservation of impulse causes new solid formations to turn, especially when the number of collisions between them is consistently increasing, into orbital motion around the star instead of radial and then chaotic motion of solid fragments in the spherical zone formed after the frontal collision (causes violating the central symmetry in a rarefied gas-dust cloud, we have not yet been considered). This spherical layer, in which the chaotic motion of solid fragments occurs, and where their orbital motion is formed, will constitute a zone of planet formation. Moreover, as will be shown in the next chapter, the shape of this zone will not in reality be spherical, but elongated, which corresponds to the nuances discussed in the new astronomical observations of planet formation.

The description of the processes of planet formation, on which the internal structure of the planets directly depends, is not included in this work. A separate article will be devoted to it. However, generally the fact of head-on collision of two compacted dust zones and the chaotic nature of the movement of heavy fragments in the very dense dusty zone of planetary formation create completely new conditions for planetary formation associated with new mechanisms. New physical effects and the activation of accretion processes will give life to new opportunities for the formation of planets that were not previously taken into account, which will help to draw attention to the theoretical possibility of the existence of new structural features of the emerging planets.

In the described scheme of formation of the planetary belt of a star, no reasons for the emergence of the predominant direction of the orbital rotation of the resulting solid fragments still, as in all previously created models, appear (unless we consider the effects external to the gas-dust cloud). It is quite difficult to find the cause of this effect for isolated system; it is not visible at all. An unconventional solution is required. One of the possible mechanisms will be discussed in the next chapter.

Modern models suggest that almost all the dust of the protoplanetary cloud is consumed in the formation of planets and asteroids. At the same time, from astronomical observations it follows that the final mass of the already formed planetary system of the evolved star system is very small in comparison with the initial dust reserves in the gas-dust cloud. For example, for a solar system, where the Sun is a typical star, the mass of matter not included in the Sun and constituting the planetary system of the Sun, is about $0.03 \%$ of total mass. Moreover, the planetary system includes not only heavy elements, but also gas. Considering that, as already mentioned above, about $1 \%$ of the mass of the gas-dust cloud usually consists of heavy elements, the planets and asteroids are created only from a very small residue from the original dust. Accordingly, in the modern approaches to the issue of planetary formation, by the time the processes occurring in the protoplanetary disk reach their peaks there probably should be a small amount of dust compared to the content in the original cloud. Modern models of planetary formation do not even attempt to explain the current situation with the mass balance and the efficiency of the process of planet formation with such a low dust content within the protoplanetary disk. This can be said not only about the efficiency of the accretion processes, but also about the problems of separation of the heaviest chemical elements in the processes of the formation of protoplanets.

If we return to the considered scheme of a self-compressing rarefied gas-dust cloud, then, considering that most of the dust at the moment of ignition of the star falls on the peripheral zones, we can assume that the mass of the resulting heavy nucleus of a single star is comparable in order of magnitude to the mass of its planetary system. Even if this is not the case, it should not be the remains of dust that participate in the accretion processes during the formation of the planetary belt, most of which is already 
absorbed by the star before the beginning of the formation of the planets, as is considered today, but almost all the dust of the original cloud concentrated in the zone of the emerging planets.

Taking into account that the main part of the dust belongs to the peripheral zone, which transmits a mechanical impulse in the radial direction to the star, then all the failed planets after the initial stage of formation should effectively and quickly be absorbed in the form of asteroids by the star.

\section{Preliminary Schematic Estimates of the Possibility of Degeneration of the Structure of the Solid Core of a Star and the Reasons for the Appearance of the Directional Rotation of the Substance of a Single Star System}

The question of the possibility of degeneration of a structure requires a rigorous approach and is unsolvable in the framework of a generalized analytical approach. However, a rigorous approach in this case is so multifactorial and laborious that it requires collective labor. But the collective study of the scientific question is always associated with large financial costs. Therefore, it is rational to make at least a generalized assessment of the attractiveness of starting work in this direction.

For the formation of a degenerate structure, a strong increase in pressure is required in the spatial zone of the substance, which must become the degenerate structure. In the theory of the evolution of stars, only the variants of the increases in pressure in the studied zone solely due to gravity depending on the mass of the star core are considered (about the degenerate structures, for the evolution of stars and the black hole variant see [3]). It is clear that for a proto-star its gravitational energy is insufficient for the degeneration of its internal structure. It is not enough, even if we take into account that in this new approach to the formation of the inner zone of a gas-dust cloud, the energy of the gravitational field of the entire gas-dust cloud as a whole is added to accelerate falling dust particles on newly protostar, i.e., the kinetic energy of particles, is characterized not by the laws of thermodynamics, but by the laws of Newtonian mechanics.

Before describing the third stage of the Kelvin compression of a rarefied gas-dust cloud (which coincides with the stage of the final formation of the structure of its core) in more detail, it is useful to specify the second stage a little. Therefore, a short summary and review of the above is required.

At the initial stage of compression of a rarefied gas-dust cloud, it is important to consider it consisting of two subsystems: dust and gas. The division of the system into two subsystems predicts the appearance of a solid nucleus washed by gas in the centre of mass of a cloud.

Subsequently, the possibility of dividing the gas and dust system into gas and dust subsystems is not only preserved, but continues to be necessary. The heavy particles that continue to approach from the periphery do not have averaged energy after mutual elastic collisions with the particles around them, they cannot be tied to statistical laws that increase the pressure in the central zone, as in the case of gas particles, they carry the total gravitational energy of the field in the form of kinetic energy created by the gas and dust components of the system. Their forward motion will be slowed in head-on collisions with turned around and approaching dust particles with an approximate conservation of radiality in motion, as well as distorted by collisions with gas particles. However, the general direction towards the centre will remain unchanged. Only with such a prehistory the heavy particles arriving to the central zone will, firstly, have higher kinetic energy compared to any particles of the central zone, and, secondly, not have a strict direction towards the centre of mass of the system. This means that instead of head-on collisions between the former central solid core and heavy particles moving from different sides into the central zone, a dense central vortex cloud will form around the already existing solid core. Moreover, the "pressure" parameter for such a central complex will be fundamentally inapplicable, and the "directional pressure" towards the center of mass will be determined not by the mass of the solid core and the masses of adjacent pseudo-orbital particles, but by the kinetic energies of the heavy particles falling onto the core. Furthermore, the gas that is later displaced by the arriving particles takes part in the formation of the kinetic energy of the arriving heavy particles. This is the final picture of the second stage of compression.

However, there is another feature that has not yet been discussed. Due to the transformation of the gas surrounding the central zone into plasma, the heavy particles re-entering the central zone will arrive positively charged and create electric and magnetic fields around it. The electrons that they have lost will concentrate together with the plasma electrons in the transition zone from the plasma to the region with a vortex structure, in which the directions of the swirls of individual protons and positively charged solid particles are spatially equiprobable, and the frequency of mutual collisions of rotational particles is spherically symmetrical. Beyond the zone of increased electron concentration in the plasma, the adjacent ionized hydrogen (protons) zone will begin to form in the external direction, i.e., a blurred electrical double layer will begin to form.

With the spontaneous commencement of single thermonuclear reactions in the proton layer of the plasma cloud, conditions can be created for a sharp burning out of the protons of this layer in a moderately powerful thermonuclear explosion. Given the uneven density, pressure and concentration of protons in the plasma in the radial directions, as well as a high rate of release of energy, 
a thermonuclear explosion must inevitably be accompanied by the appearance of spherical shock waves scattering from the explosion in radial directions to and from the center of mass of the cloud.

The energy of a thermonuclear explosion together with the currently accumulated energy in the central zone of accretion and the kinetic energy of the particles in the vortex zone, making a significant energy contribution, may well be enough for a qualitative jump: the core of the cloud will turn into a degenerate structure. Furthermore, not the transition of a substance into a degenerate state will initiate a thermonuclear explosion, but on the contrary, a thermonuclear explosion will induce the transition. The fact is that during the formation of a white dwarf and a neutron star, under the influence of gravitational forces, the structure degenerates first, and only as a result a thermonuclear explosion is initiated, which forms a shock wave, all of the energy from the explosion is expended on the scattering of matter. In the case being considered, at the last stage of the formation of the core of the gas-dust cloud, a thermonuclear explosion must first occur, and only then, as a result of the impact of the shock wave, the solid matter and the adjacent accretionary vortices will transfer into a single formation with a degenerate structure, accompanied by the emission of accumulated dust in the radial directions from the centre. It is in this situation that a proto-star will not slowly manifest itself in infrared radiation, but will flash in the infrared spectrum before a gradual ignition of a star, as is the case in real observations.

Two very significant factors are indicative of this alleged event. The first factor is the presence of strong magnetic fields in single stars of gas-dust clouds, and the second is the possibility of explaining the reason for the spin of the planetary zone of the cloud.

The fact is that the magnetic induction of an electrically charged rotating object is inversely proportional to the square of its size. Therefore, the sharp compression of a solid structure to a structure with a degenerate electron or neutron gas makes it possible to understand the cause of the occurrence of a strong magnetic field and create a theoretical scheme for the magnetic field generator of a star. Moreover, the magnetic field of the star is dipole, has two poles, and its magnetic field axis is skewed from the axis of rotation of the star.

Where does the directional rotation of the star come from? After all, at all stages of compression of a gas-dust cloud at rest there are no such phenomena that could break the spatial symmetry of the processes taking place. There are no obvious reasons for purposefully creating the directional mechanical rotation of the star.

When answering this question, the scheme of formation of a heavy nucleus in the centre of a gas-dust cloud considered in the present work comes to the rescue. Asymmetry can occur only at the stage of appearance of electric charge in the vortex flows falling into the centre. Then light deviations from the symmetry in the vortex flows will immediately lead to a quite pronounced directivity of the external magnetic field, which, along its lines of force, builds solid charged particles ionized by plasma falling from the peripheral region onto the central zone, which, in turn, increases the non-uniformity of the vortex flows and stimulates their alignment directly parallel to each other (positive feedback). After a certain time, a directed vortex motion will appear in a dense central vortex cloud, gradually transforming into rotation of compacted adjacent outer layers in which ionization of heavy particles occurs. A steady mechanical rotation stabilized by a magnetic field will occur. However, stabilization can never be realized and maintained instantly. Due to the precedence of the formation of a magnetic field, changes in which correct the movement of charged particles already as a result of these changes, the directions of the axes of the magnetic field resulting from the average moment of the mechanical rotation of the vortex zone and the mechanical rotation of the adjacent plasma layers in the steady state will always be close, but will not coincide. The new formation with a degenerate structure that arises after a thermonuclear explosion must preserve the prevailing prehistory with the directions of the axes. Not only is the appearance of a large magnetic field in a single star, but also the explanation of the deviation of the axes of mechanical rotation and magnetic field are very important factors in favour of the hypothesis of substance degeneration in the core of the future nucleus of a single star.

Therefore, it is most likely that the axial rotation of the star and, as a consequence, the first signs of orbital motion of the matter, pulled towards the central zone of the gas-dust cloud, originate in the second stage of compression of the rarefied gas-dust cloud. It is at this stage that spherically symmetric heavy particle flows in the central compacted zone of the gas-dust cloud and its surroundings are first formed, transforming over time into directional flows of electrically charged heavy particles, rotating the adjacent plasma zones and violating the spherical symmetry of the plasma cluster.

A subsequent thermonuclear explosion in the inner zone of a gas-dust cloud with a broken central symmetry of the proton distribution, which characterizes the beginning of the third stage of compression of a rarefied gas-dust cloud, will stretch the inner compacted zone of the gas-dust cloud, the particles of which, prior to the explosion, already have some preferential direction of the orbital velocity components. This predominant direction of the velocity components will subsequently, after a collision with the peripheral compacted zone, be transmitted to all elements of the dust subsystem of the formed planet formation zone. For this reason, by the time of the emergence of planets, the considered zone of planet formation will inevitably turn into a flat disk in which the planets will begin to rotate in orbits with a predominant direction of rotation. The mechanism of the emergence and formation of the directed 
orbital motion of the planets requires special modelling and cannot, at this time, be perceived as an indisputable consequence of the thermonuclear explosion in the core of the cloud. However, the exposed physical principles of the compression process of a rarefied gas-dust cloud quite clearly and convincingly signify in favour of this event.

From the presented material it follows that the hypothesis of degeneration of the solid core of a gas-dust cloud and the spread of heavy elements of the inner zone by a shock wave of a thermonuclear explosion in radial directions while maintaining the moments of their rotational motion is very attractive, very relevant and requires special study.

\section{A little about the Solar System}

Our Sun is not the first-generation star and was formed from a gas-dust cloud. Most often, stars are born in groups, and their birth occurs in huge gas and dust clusters of equatorial galaxy sections. Astronomers observe many so-called "star associations", which probably arose at a certain stage in the evolution of the Universe as a result of explosions of superstars formed in massive, not gas, but gas-dust clouds. The stars resulting from the explosion of superstar that have high initial velocities are scattered, and the remaining stars with small initial velocities form an open star cluster. Open star clusters are not absolutely stable and eventually lose their stars, forming a "star trail".

Despite the fact that the Sun according to the characteristics of its movement can enter into different observable star "tails" of stars ejected from clusters, our star was rarely included into any star accumulation, and, most probably, was born as a single star. In star clusters, stars are so close to each other that they destroy their circumstellar planetary systems. But our solar system is quite unique and shows us its planetary system, including the asteroid belt, which is beyond the orbit of Pluto, and comet clouds. Four exterior relative to Earth planets are largely composed of hydrogen and helium. But beyond the orbit of Neptune are trans-Neptune objects consisting of frozen water, ammonia and methane, and the asteroid belt between Mars and Jupiter is similar in composition to the terrestrial planets because it consists of silicates and metals.

Currently it is impossible to explain such complex planetary system of the Sun in terms of compression of a gas-dust cloud within the model of compression of dusty gas, and not compression of a gas-dust cloud consisting of two coexisting and relatively independent subsystems.

Another theoretical aspect should be noted for the solar system. Almost the entire total moment of the momentum of the solar system lies in the movement of planets, which have a small total mass in comparison with the mass of the Sun. For this reason, it appears that the planets were not the product of the evolution of an isolated solar system.
Nevertheless, all the characteristics of the planetary movement, their composition and division into groups unequivocally indicate a single and simultaneous process of the formation of planets inside the solar system. It is quite difficult to give a comprehensive explanation of this within the framework of the existing knowledge about the evolution of a single star system. This work should help provide an incentive for a better understanding of the issue.

As follows from the material presented above, the heavy core of the Sun was formed even before the Sun ignited as a star. With increasing core temperature, the lighter components evaporated from it. The fact that the spectrum of the Sun does not indicate high concentrations of heavy elements in the composition of the star does not prevent the Sun from having a heavy core. Whether the core of the Sun is a degenerate structure is a matter of future scientific research. The strong magnetic field of the Sun does not prove but allows for the assumption that the presence of a degenerate structure in the Sun's core is most probable. Moreover, the presence of a degenerate structure in the core of the Sun guarantees the complete absence of a flow of heavy elements from the core (while the existence of the dense core itself cannot be doubted) to the periphery, which is consistent with observations (with the interpretation of the Sun spectrum). The discrepancy between the axes of the mechanical rotation and the magnetic field is also an indirect sign of the presence of a degenerate structure. Another indirect indicator to the birth of the Sun as a single star that forming the core of a degenerate structure is a planetary disk with a complex spatial and structural configuration, with a complex distribution of types of matter.

\section{The Main Points of the Material Presented (Highlights)}

In this paper, the analysis is based on "common sense" (according to the methodology of "preliminary fitting of mechanics"). It is shown that the formation of a single star system occurs at the expense of spontaneous gravitational contraction of a calm rarefied gas-dust cloud characterized by the following features:

- the gas-dust system needs for a calm rarefied gas-dust cloud to be divided into two sub-systems: gas and dust;

- $\quad$ it is unacceptable to consider a calm rarefied gas-dust cloud as a gas cloud;

- at the initial stage of the Kelvin compression of a rarefied gas-dust cloud heavy particles do not have the advantage of moving to the center;

- in a quiet rarefied gas-dust cloud, at the initial stage of its compression, the gas and dust subsystems are practically independent of each other and should manifest themselves as pure clouds; 
- $\quad$ the dust subsystem is characterized not by pressure, but by the kinetic energies of its constituent particles;

- dust is stratified into two compacted zones: internal and peripheral;

- $\quad$ the formation of a heavy solid core in a gravitationally contracted rarefied and calm gas-dust cloud is inevitable;

- the bulk of the dust is contained in the peripheral zones;

- $\quad$ the accumulation of dust on the periphery will occur not due to accretion, but due to acts of collisions of dust particles having a radial component of their speeds as the predominant one;

- although the outflow of dust in the central zone retains its activity, it also turns into a discrete zone of increased dust density, primarily due to the influence of a usual geometric factor in the spatial distribution of the cloud;

- the average statistical result will bring about a formation of a vortex compacted zone of heavy particles, falling on the seeding core;

- $\quad$ plasma is formed in the central area of the cloud;

- the launch stage of self-regulating thermonuclear reactions (i.e., the star ignition stage) and the appearance of the stellar wind is preceded by a small thermonuclear explosion, accelerating the main part of the solid particles of the inner zone towards the periphery;

- the complex composite structure of the central zone (nucleus and vortex zone of accretion) will undergo a transformation, having turned into a degenerate structure;

- two compacted dust zones undergo a head-on collision with each other;

- $\quad$ almost all the dust of the original cloud is involved in the accretion processes during the formation of the planetary belt;

- the appearance of a large magnetic field in a single star and the deviation of the axes of mechanical rotation and the magnetic field is due to the transformation of the complex structure of the central zone into a degenerate structure;

- $\quad$ the appearance of directional rotation of the planetary system is also due to thermonuclear explosion.

\section{Conclusions}

This paper shows that analysis at the level of common sense in a logical chain based on physical principles, in certain cases, has unique power. Where common sense is applicable, the directions in which it is required to move forward with the further construction of the physical model become clear, and after the development of the current version of the model the possibilities of assessing the correctness of the resulting version appear. The lack of attention to the mechanistic approach in the natural sciences is a negative phenomenon that must be avoided.

In the framework of this work, the mechanistic approach revealed very important new nuances of the evolution of a single stellar system. It was shown that a single star of a non-first generation must have a heavy core. It was found that the dust component of a rarefied gas-dust cloud in the process of gravitational compression of the cloud splits into two spherical zones, accumulating heavy elements. These processes are the essence of the initial stage of the separation of heavy elements involved in the formation of planets. The subsequent head-on collision of these zones forms the belt of formation of planets and asteroids, completing the course of separation of heavy elements in the processes of active accretion. At the same time, a set of new, not previously considered opportunities for the formation of planets is becomes available in the zone of planet formation. However, in the framework of this work, the set of new opportunities is not discussed, only the reasons why new physical effects should arise are indicated.

In addition, the paper attempts to explain the appearance of the axial mechanical rotation of the star and the dipole magnetic field, whose axis does not coincide with the axis of rotation of the star. It was suggested that the heavy core of a single star has a degenerate structure. An explanation is given of the possible reason for the emergence of a common direction of rotation for the entire planetary system that is not related to external influence (and external energy expenditure) on the gas-dust cloud.

It is possible that stars of stellar associations, in contrast to single stars, do not have strong magnetic fields. It is possible that the stars of star clusters do not have circumstellar planetary systems not because of their destruction during the close passages of other stars in the cluster, but because they never had them in the first place.

\section{REFERENCES}

[1] D K Prialnik. An Introduction to the Theory of Stellar Structure and Evolution, Second Edition, Cambridge University Press, 2010.

[2] A. S. Belyaev. Gravitational Contraction of a Dust Cloud in Space, Universal Journal of Physics and Application, Horizon Research Publishing (HRPUB), Vol.10, No 1, 22-30, 2016. Online available fromhttp://www.hrpub.org/d ownload/20160331/UJPA5-18406009.pdf.

[3] A. S. Belyaev, Trends of stellar evolution in modern physics. Universal Journal of Physics and Application, Horizon Research Publishing (HRPUB), vol. 11, No 6, 247-263, 2017. Online available from http://www.hrpub.org/downloa d/20171230/UJPA5-18410784.pdf.

[4] A. S. Belyaev. Photon gas, Universal Journal of Physics and Application, Horizon Research Publishing (HRPUB), 
Vol.10, No.1, 11-15, 2016. Online available from http://www.hrpub.org/download/20160229/UJPA3-184060 08.pdf.

[5] A. S. Belyaev, A new model of the birth of the Universe. Universal Journal of Physics and Application, Horizon Research Publishing (HRPUB), vol. 11, No 5, 182-189, 2017. Online available from http://www.hrpub.org/downloa d/20170930/UJPA7-18410201.pdf.

[6] A. S. Belyaev, Accounting for the Geometric Factor in Classical Gravity. Frontiers of Astronomy, Astrophysics and Cosmology, Science and Education publishing (SciEP), vol. 4, No 1, 18-29, 2018. Online available from http://www.sciepub.com/faac/abstract/9838

[7] A. S. Belyaev, the Possibility of Spontaneous Gravitational Contraction of a Gas Cloud in Outer Space with Simultaneous Cooling of the Cloud. Universal Journal of Physics and Application, Horizon Research Publishing (HRPUB), vol. 12, No 2, 24-29, 2018. Online available from http://www.hrpub.org/download/20180530/UJPA2-184114 01.pdf. 